\title{
The Effect Of Intraoperative Use Of
}

\section{Dexmedetomidine During The Daytime Operation Vs The Nighttime Operation On Postoperative Sleep Quality And Pain Under General Anesthesia}

This article was published in the following Dove Press journal:

Nature and Science of Sleep

\section{Bijia Song \\ Yang Li \\ Xiufei Teng \\ Xiuyan Li \\ Yanchao Yang \\ Junchao Zhu}

Department of Anesthesiology, Shengjing Hospital of China Medical University, Shenyang, Liaoning, People's Republic of China
Correspondence: Junchao Zhu

Department of Anesthesiology, Shengjing Hospital of China Medical University,

Shenyang, Liaoning, People's Republic of China

Email zhujunchaol@hotmail.com
Objectives: The aim of our study was to compare the effect of using dexmedetomidine (DEX) during the daytime operation or the nighttime operation under general anesthesia on postoperative sleep quality and pain of patients.

Methods: Seventy-five patients scheduled for elective laparoscopic abdominal surgeries under general anesthesia were randomly assigned to receive operation in the Day Group (8:00-12:00) and the Night Group (18:00-22:00). The Portable Sleep Monitor (PSM) was performed on the following 3 nights: the night before surgery (Sleep 1), the first night after surgery (Sleep 2), and the third night after surgery (Sleep 3). Postoperative pain scores using visual analogue scoring scale, subjective sleep quality using the Athens Insomnia Scale, total dose of general anesthetics and PCA pump press numbers were also recorded.

Results: Intraoperative administration of DEX for patients in the Day Group could improve sleep quality with a higher sleep efficiency and a lower AIS subjective sleep quality than patients in the Night Group at Sleep 2 ( $\mathrm{P}<0.001$ and $\mathrm{P}=0.001$, respectively) and Sleep 3 ( $\mathrm{P}$ $<0.001$, respectively). There were marked lower rapid eye movement (REM) sleep and Stable sleep in the Night Group than that in the Day Group at Sleep 2 ( $\mathrm{P}<0.001$ and $\mathrm{P}=$ 0.032, respectively) and Sleep $3(\mathrm{P}<0.001$, respectively). Patients in the Day Group have better pain relief and less PCA pump press numbers than patients in the Night Group.

Conclusion: Using dexmedetomidine during the daytime operation can better improve postoperative sleep quality and pain than nighttime operation in patients undergoing laparoscopic abdominal surgeries.

Keywords: dexmedetomidine, sleep quality, general anesthesia, postoperative pain

\section{Introduction}

General anesthesia is a medically induced state of low reactivity consciousness which is similar to natural sleep. ${ }^{1}$ Some studies found that general anesthesia as an independent risk factor could result in a desynchronization of the circadian time structure and cause postoperative sleep disorders characterized by reduced rapid eye movement (REM) and slow wave sleep (SWS), ${ }^{2}$ which have significant deleterious impacts on postoperative outcomes, such as postoperative fatigue, severe anxiety and depression, emotional detachment and delirium, and even pain sensitivity or postoperative pain of patients. ${ }^{3,4}$ Clinical trials have already proved that intraoperative use of dexmedetomidine (DEX) for general anesthesia, a highly selective 
alpha-2 adrenergic agonist, was able to improve sedative and analgesic effects, reduce the incidence of postoperative delirium and promote sleep quality. ${ }^{5-10}$ However, Tan et al reported that with the deeper sedative state provided by DEX in the daytime, the elderly male patients undergoing TURP under spinal anesthesia suffered worse sleep on the night of surgery. ${ }^{11}$ Thus, what the effect of intraoperative using DEX at different time periods under general anesthesia on postoperative sleep quality and pain will be needs further study. Based on these considerations, we sought to answer two questions in this study:

1. What is the effect of using DEX during daytime operation or nighttime operation under general anesthesia on postoperative sleep quality of patients?

2. What is the difference between intraoperative using DEX at different time periods on postoperative pain of patients under general anesthesia?

\section{Materials And Methods}

This study was approved by Human Research Ethical Committee of Shengjing Hospital, Shenyang, Liaoning Province, China (IRB registration number 2017PS29K) and was compliant with the Declaration of Helsinki. Written informed consent was obtained from all subjects participating in the trial. The trial was registered prior to patient enrollment at clinicaltrials.gov (NCT03990987).

\section{Participants}

Patients who underwent elective laparoscopic abdominal surgeries under general anesthesia at Shengjing Hospital of China Medical University were enrolled in this study if they met the following inclusion criteria: age between 30 and 55 years and American Society of Anaesthesiologists (ASA) physical status I or II. Exclusion criteria included cardiovascular disease, long-term use of analgesic, preoperative heart rate (HR) less than 50 beats/min, second- or third-degree atrioventricular block, use of sleep-promoting medications, sleep disorder, sleep apnea syndrome, history of abnormal operation or anesthesia recovery, psychosis or a patient with a language communication disorder, did not provide informed consent.

\section{Sample Size}

Sample size calculation was based on the primary outcome of subjective sleep quality between the two groups. According to our preliminary study, the most difference of subjective sleep quality in the two groups was 0.8 . So the estimated variability we chose was 0.8 between the two groups, and the standard deviation was 1.1; then we calculated the size of the sample, and twenty-nine patients for each group were required, assuming a two-sided Type I error $(\alpha)$ of 0.05 and a power of $80 \%$. Potential loss was expected during follow-up or due to drop out; therefore, a total of 75 patients were enrolled in this study.

\section{General Anesthesia}

Before the operation, all patients fasted for 8-12 hrs. Upon arriving in the operating room, standard monitoring, including an electrocardiogram, non-invasive blood pressure (NIBP), heart rate (HR) and peripheral oxygen saturation $\left(\mathrm{SpO}_{2}\right)$, were recorded using a multifunctional monitor (Draeger Infinity Delta; Draeger Medical System Inc., Luebeck, Germany). General anesthesia was induced with propofol $(2 \mathrm{mg} / \mathrm{kg})$, sufentanil $(0.2 \mu \mathrm{g} / \mathrm{kg})$ and cisatracurium $(0.15 \mathrm{mg} / \mathrm{kg})$. After adequate jaw relaxation was attained, tracheal intubation was performed, and each patient was mechanically ventilated with a tidal volume and ventilation rate adjusted to maintain the pressure of end-tidal carbon dioxide (ETCO2) at 35-45 $\mathrm{mmHg}$. Intravenous infusion was switched to a maintenance syringe pump at rate of $100 \mu \mathrm{g} / \mathrm{kg} / \mathrm{min}$ for propofol, $0.15-0.2 \mu \mathrm{g} / \mathrm{kg} / \mathrm{min}$ for remifentanil and $0.2-0.7 \mu \mathrm{g} / \mathrm{kg} / \mathrm{h}$ for DEX, and together with inhaled $50 \%$ air and $50 \%$ oxygen $\left(\mathrm{O}_{2}\right)$ at a fresh gas flow rate of $2 \mathrm{~L} / \mathrm{min}$. Cisatracurium $(0.05 \mathrm{mg} / \mathrm{kg})$ was intermittently used for muscle relaxation. The anesthesiologist adjusted the intravenous speed of remifentanil and DEX according to hemodynamic parameters and BIS (BIS monitor; Aspect Medical System, Newton, MA). The BIS was maintained between 40 and 55 during the operation. Ten minutes before the end of the surgery, all anesthetic infusions were stopped, and after extubating, the patients were transferred to the PACU for continuous monitoring.

\section{Study Protocol And Measurements}

Seventy-five patients were assigned to the Day Group (D Group, 8:00-12:00) $(\mathrm{n}=37)$ and the Night Group (N Group, 18:00-22:00) $(\mathrm{n}=38)$, using a computer-generated randomized table. Patients received propofol, remifentanil and DEX for general anesthesia maintenance. After the operation, the PCA system was attached, and the patients were instructed in its use. The PCA system (sufentanil 100 $\mu \mathrm{g}$ diluted into $100 \mathrm{~mL}$ ) was programmed to deliver a continuous dosage of sufentanil $0.02 \mu \mathrm{g} \mathrm{kg}^{-1} \mathrm{~h}^{-1}$ and a bolus dose of $0.02 \mu \mathrm{g} / \mathrm{kg}$, with a 10 -mins lockout interval. 
Finally, the patients were discharged from PACU to quiet ward where NIBP, SPO2 and HR were monitored.

BIS, mean arterial pressure (MAP) and HR of each patient were recorded at 5 mins after entering the operation room (T0); intubation (T1); 5 mins after intubating (T2); at the end of the operation (T3); extubation (T4); 5 mins after extubating (T5). And the total dose of remifentanil and DEX of both groups during the operation was recorded.

The Portable Sleep Monitor (PSM, PSM100A; Sealand Technology (Chengdu) Co. Ltd) was performed on the following 3 nights from 23 PM to 6 AM: the night before surgery (Sleep 1), the first night after surgery (Sleep 2) and the third night after surgery (Sleep 3). Sleep variables, such as sleep efficiency (the ratio of total sleep time/total recording time), the Athens insomnia scale (AIS) subjective sleep quality, the percentage of REM sleep, Unstable sleep and Stable sleep were recorded and analyzed by sleep laboratory staff who was blinded to patients' information. Postoperative pain scores were evaluated by Visual analogue scale (VAS) score, ${ }^{12}$ which indicated that 0 was considered painless, and a score of 10 was considered as intense pain. Scores of 4 and below were considered mild pain (pain does not affect sleep), and scores of 5 to 6 were considered moderate pain (pain influences sleep, but the patients can still fall asleep). Scores of 7 and above were considered severe pain (due to the pain, the patient is unable to sleep or wakes up). Patients were encouraged to push the analgesic-demand button when they experienced significant pain (VAS $>4$ at rest). VAS was measured at 1, 6, 24, $48 \mathrm{hrs,} \mathrm{postopera-}$ tively. Adverse effects such as hypotension, bradycardia, nausea and vomiting and hypoxemia were also treated accordingly.

\section{Statistical Analysis}

SPSS 20.0 statistical software (SPSS, Inc., Chicago, IL, USA) and GraphPad Prism 6.0 software were used for data analysis. The Kolmogorov-Smirnov test was used to assess the distribution of variables. Continuous data were analyzed by using independent-samples Student's $t$-test and represented as mean \pm standard deviation. For statistical analysis of sleep measures, AIS and sleep efficiency, independent-samples Student's $t$-test and the Wilcoxon rank-sum test were used. One-way analysis of variance (ANOVA) followed by Bonferroni correction was used for multiple comparisons. $P<0.05$ was considered to be statistically significant.

\section{Results}

We initially assessed 93 patients for eligibility to participate in our study (Figure 1); of these, 12 patients refused to participate, 6 patients did not meet the inclusion criteria and the remaining 75 patients enrolled to the study. Following the completion of the study, 7 patients in the D Group were excluded from the study: 3 patients failed sleep monitoring due to detachment of electrode, 3 patients failed sleep monitoring due to allergic reaction to electrode paste and 1 patient received rescue analgesia. Eight patients in the $\mathrm{N}$ Group were excluded from the study: 4 patients failed sleep monitoring due to detachment of electrode, 2 patients failed sleep monitoring due to allergic reaction to electrode paste and 2 patients received rescue analgesia. Finally, the data from 30 patients in the D Group and 30 patients in the N Group were analyzed in the present study.

\section{General Conditions Of The Study Groups}

There were no statistically significant differences $(\mathrm{P}>$ 0.05 ) for age, weight, duration of anesthesia (mins), duration of surgery (mins) and estimated blood loss (mL) among the study groups. However, the extubation time of patients in the $\mathrm{N}$ Group was longer than that of patients in the D Group ( $<$ 0.001) (Table 1).

\section{Anesthesia-Related Information Duration The Operation}

Patients in the N Group had statistically lower BIS values than those in the D Group from T1 to T5 $(46.43 \pm 2.6$ vs $51.73 \pm 3.4,49.13 \pm 2.1$ vs $51.50 \pm 3.0,54.33 \pm 2.4$ vs 59.03 $\pm 3.3,70.57 \pm 4.0$ vs $74.77 \pm 4.1,80.10 \pm 2.8$ vs $83.17 \pm 4.0$ ) (Figure 2A). There were no significant differences with respect to the baseline MAP and $\mathrm{HR}$ at $\mathrm{T}_{0}$ between the two groups. Furthermore, we observed sharp increases in MAP and HR evoked by intubation. Subsequently, MAP and HR were maintained at lower levels than baseline to extubation. And, patients in the N Group showed obvious lower levels in MAP and HR from T1 to T5 than patients in the D Group (Figure 2B and C, $\mathrm{P}<0.05$, respectively).

\section{Intraoperative Dosage Of Remifentanil And DEX Of Study Groups}

The total dose of DEX in the N Group was significantly less than that in the D Group $(55.63 \pm 7.2$ vs $61.91 \pm 11.3$ ) (Table 2, $\mathrm{P}=0.013$ ). And the total dose 


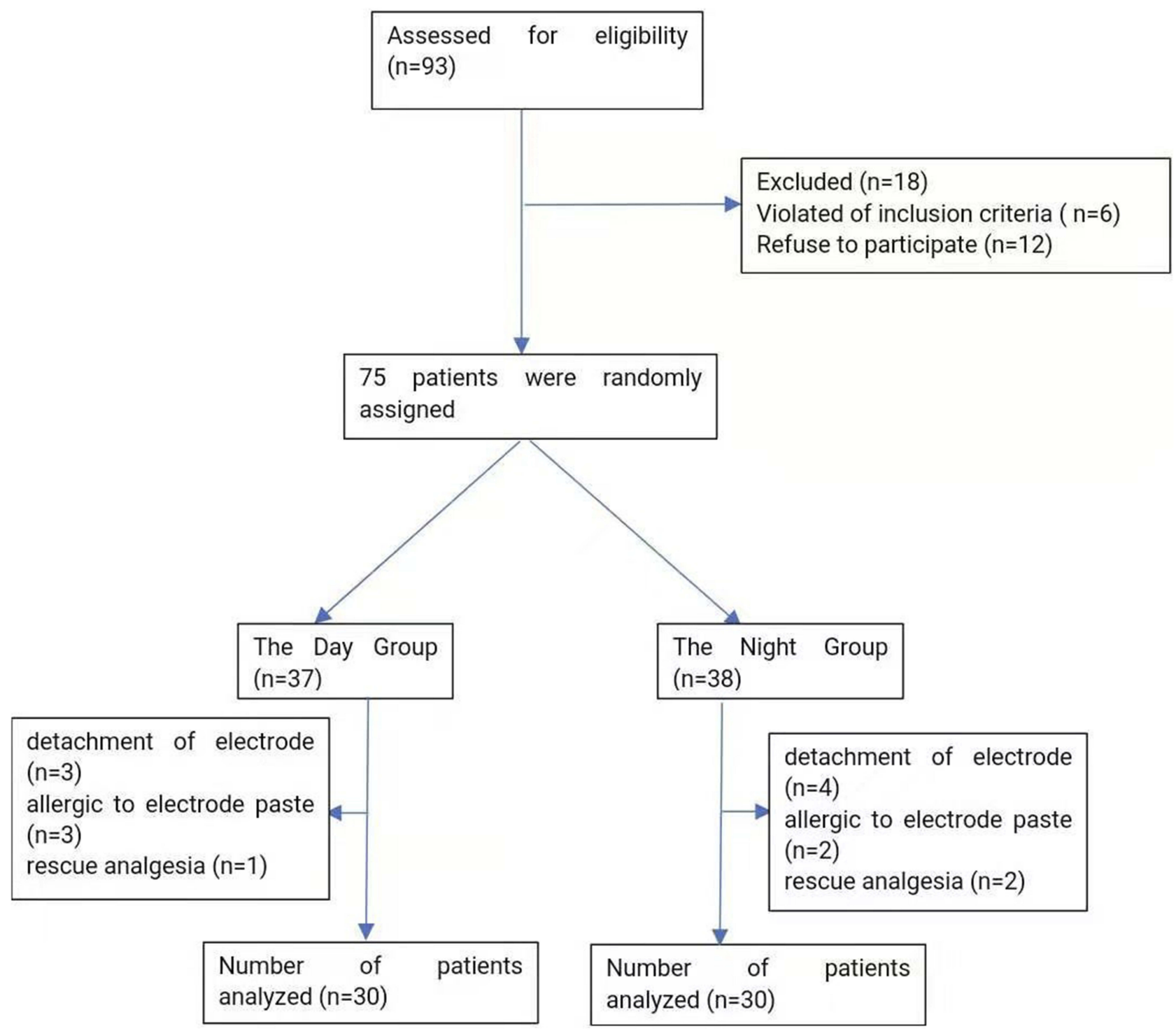

Figure I Flow-chart showing included and excluded patients.

of remifentanil in the N Group also showed signifi- Postoperative Sleep Quality

cantly less than those in the D Group $(0.50 \pm 0.1$ vs Patients suffered from obvious sleep disturbance after $0.66 \pm 0.1)$ (Table 2, $\mathrm{P}<0.001$ ). surgery. Compared to Sleep 1, a lower sleep efficiency

Table I Baseline Characteristics Of Patients In The Day Group And The Night Group

\begin{tabular}{|l|l|l|l|}
\hline & Day Group & Night Group & $\boldsymbol{P}$ \\
\hline Age (years) & $42.13 \pm 7.1$ & $43.43 \pm 6.3$ & 0.454 \\
\hline Weight (kg) & $60.47 \pm 7.5$ & $62.40 \pm 7.0$ & 0.305 \\
\hline Intraoperative data & & & \\
Duration of anesthesia (mins) & $94.13 \pm 20.1$ & $92.60 \pm 11.0$ & 0.715 \\
Duration of surgery (mins) & $88.47 \pm 20.8$ & $88.23 \pm 11.0$ & 0.957 \\
Extubation time (mins) & $7.5 \pm 1.8$ & $9.2 \pm 1.7$ & $<0.001$ \\
Estimated blood loss (mL) & $56.43 \pm 16.2$ & $58.93 \pm 12.6$ & 0.507 \\
\hline
\end{tabular}

Notes: variables were presented as mean \pm SD. 
(a)

(c)

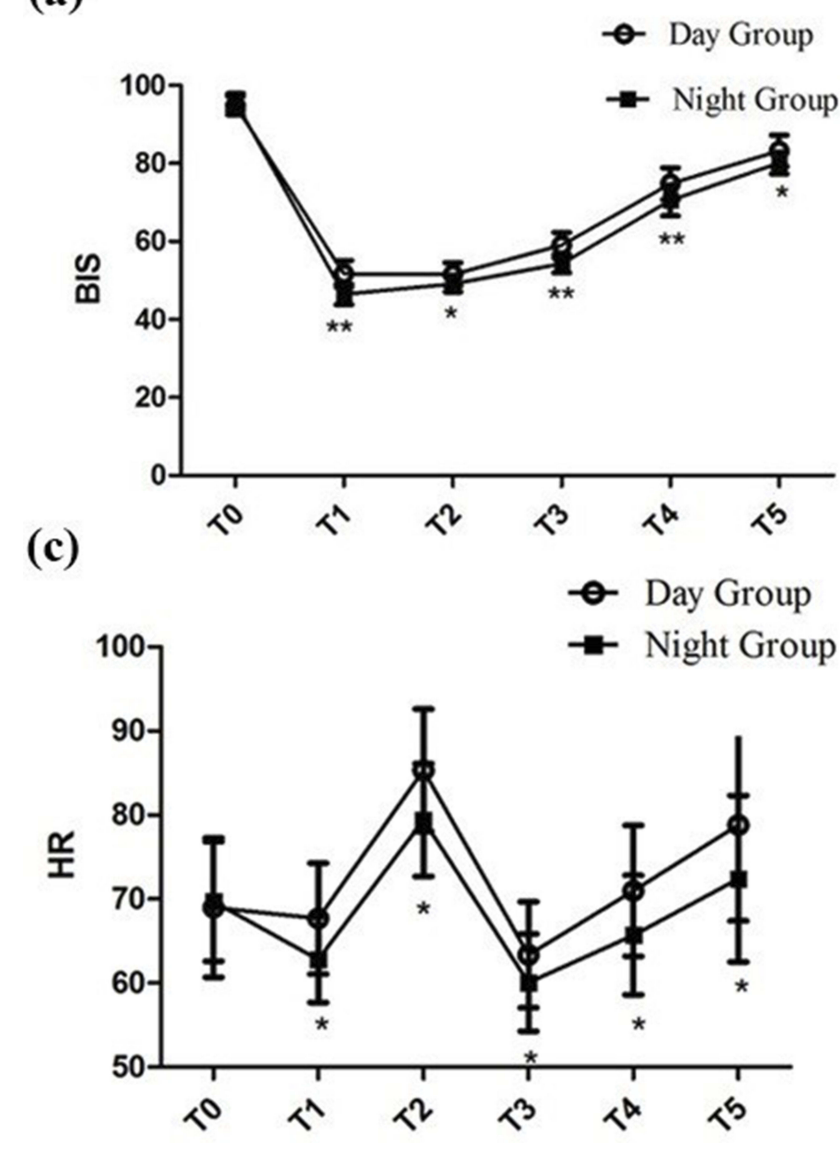

(b)

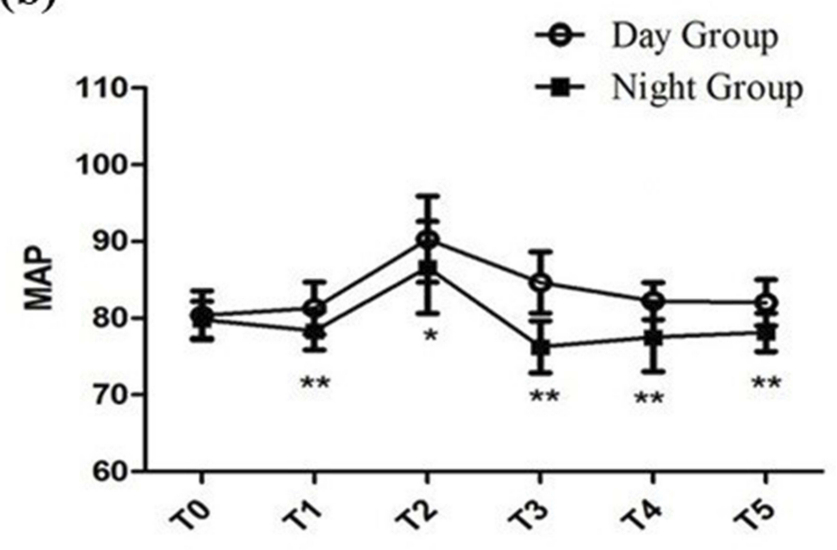

Figure 2 BIS, Heart rate (HR) and Mean arterial pressure (MAP) during the operation. (A) Intraoperative BIS at different time points; (B) intraoperative MAP at different time points; $(\mathbf{C})$ intraoperative HR at different time points; * vs the Night Group: $\mathrm{P}<0.05$; ** vs the Night Group: $\mathrm{P}<0.00 \mathrm{I}$. T0: 5 mins after entering the operation room; TI: Intubation; T2: 5 mins after intubating; T3: At the end of the operation; T4: Extubation; T5: 5 mins after extubating.

and a higher AIS subjective sleep quality were presented at Sleep $2(71.97 \pm 2.8$ vs $84.85 \pm 3.5$ and $4.63 \pm 1.0$ vs 2.23 $\pm 0.8, \mathrm{P}<0.001$, respectively) and Sleep $3(78.08 \pm 3.0$ vs $84.85 \pm 3.5$ and $3.87 \pm 0.9$ vs $2.23 \pm 0.8, \mathrm{P}<0.001$, respectively) in the $\mathrm{N}$ Group (Figure $3 \mathrm{~A}$ and $\mathrm{B}$ ). Intraoperative administration of DEX for patients in the D Group could improve sleep quality with a higher sleep efficiency and a lower AIS subjective sleep quality than patients in the N Group at Sleep $2(77.04 \pm 1.7$ vs $71.97 \pm 2.8$ and $3.73 \pm 1.0$ vs $4.63 \pm 1.0, \mathrm{P}<0.001, \mathrm{P}=0.001$, respectively) and Sleep $3(82.52 \pm 3.6$ vs $78.08 \pm 3.0$ and $2.87 \pm 0.7$ vs 3.87 $\pm 0.9, \mathrm{P}<0.001$, respectively) (Figure $3 \mathrm{~A}$ and $\mathrm{B}$ ). The

Table 2 Total Dose Of Dexmedetomidine And Remifentanil Of Patients In The Day Group And The Night Group

\begin{tabular}{|l|l|l|l|}
\hline & Day Group & Night Group & $\boldsymbol{P}$ \\
\hline Dexmedetomidine $(\mu \mathrm{g})$ & $61.9 \mathrm{I} \pm \mathrm{II} .3$ & $55.63 \pm 7.2$ & 0.013 \\
Remifentanil $(\mathrm{mg})$ & $0.66 \pm 0.1$ & $0.50 \pm 0.1$ & $<0.00 \mathrm{I}$ \\
\hline
\end{tabular}

Notes: variables were presented as mean \pm SD. sleep stages distribution is shown in Figure 4. Compared with Sleep 1, Unstable sleep time was longer at Sleep 2 $(52.05 \pm 3.2$ vs $42.35 \pm 4.2)$ and Sleep $3(46.45 \pm 2.8$ vs $42.35 \pm 4.2$ ) in the $\mathrm{N}$ Group and Unstable sleep time was also longer at Sleep 2 and Sleep 3 in the D Group than that in the N Group (57.66 \pm 4.6 vs $52.05 \pm 3.2$ and 53.94 \pm 4.3 vs $46.45 \pm 2.8, \mathrm{P}<0.001$, respectively) (Figure $4 \mathrm{C}$ ). Compared with Sleep 1 in the D Group, there were significant decreases in REM sleep and Stable sleep at Sleep $2(24.28 \pm 2.4$ vs $19.06 \pm 1.9$ and $33.38 \pm 4.6$ vs 28.89 $\pm 3.2, \mathrm{P}<0.001$, respectively) and Sleep 3 (24.28 $\pm 2.4 \mathrm{vs}$ $21.67 \pm 1.9$ and $33.38 \pm 4.6$ vs $31.88 \pm 3.1, \mathrm{P}<0.001$ and $\mathrm{P}<$ 0.05 , respectively) (Figure $4 \mathrm{~A}$ and $\mathrm{B}$ ) and there were marked lower REM sleep and Stable sleep in the $\mathrm{N}$ Group than that in the D Group at Sleep $2(15.83 \pm 1.8$ vs $19.06 \pm 1.9$ and $26.51 \pm 5.0$ vs $28.89 \pm 3.2 \mathrm{P}<0.001, \mathrm{P}=$ 0.032 , respectively) and Sleep $3(18.13 \pm 1.9$ vs $21.67 \pm 1.9$ and $27.93 \pm 5.0$ vs $31.88 \pm 3.1, \mathrm{P}<0.001$, respectively) (Figure 4A and B). 
(a)

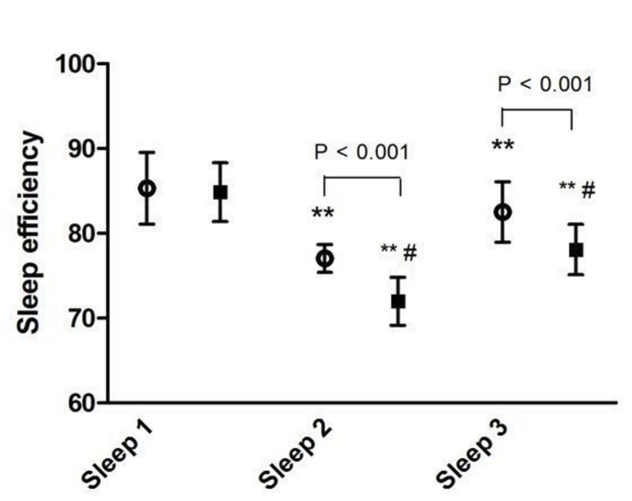

Day Group

Night Group

(b)

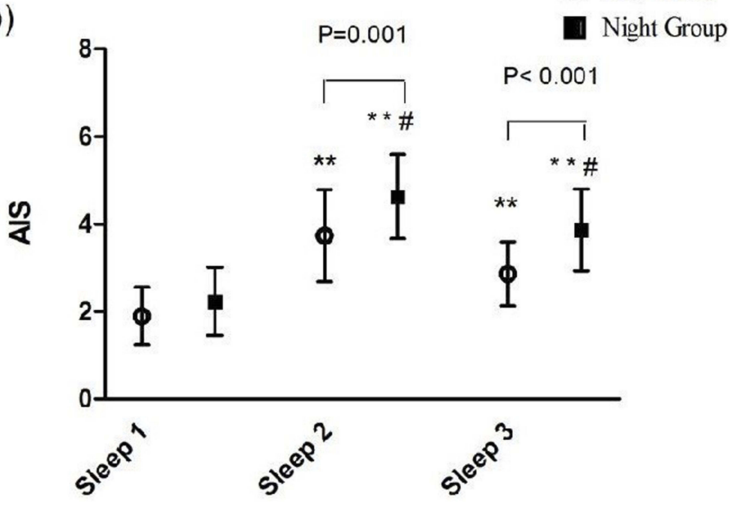

Figure 3 Comparison of sleep efficiency and the AIS scores between the Day Group and the Night Group. (A) Sleep efficiency; (B) AIS. AIS: The Athens Insomnia Scale; Sleep efficiency: the ratio of total sleep time/total recording time. Sleep I: the night before surgery; Sleep 2: the first night after surgery; Sleep 3: the third night after surgery. In the same group, ** vs Sleep I: $\mathrm{P}<0.001$. At the same point, \# vs the Day Group: $\mathrm{P}<0.05$.

(a)

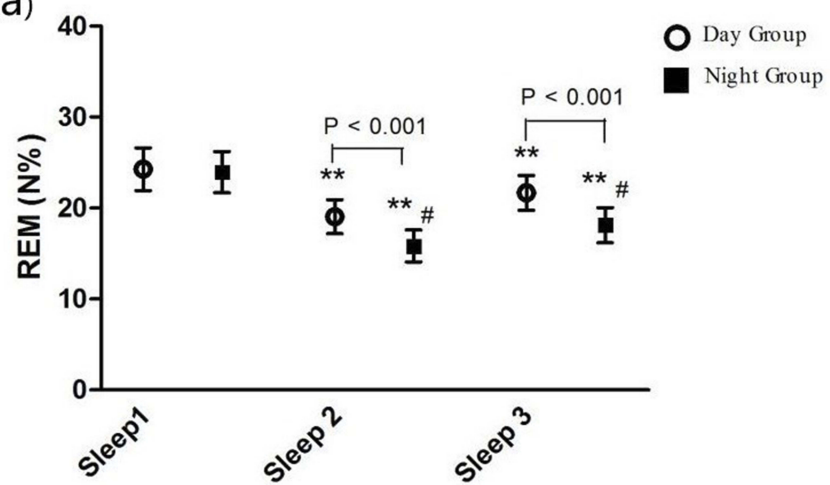

(c)

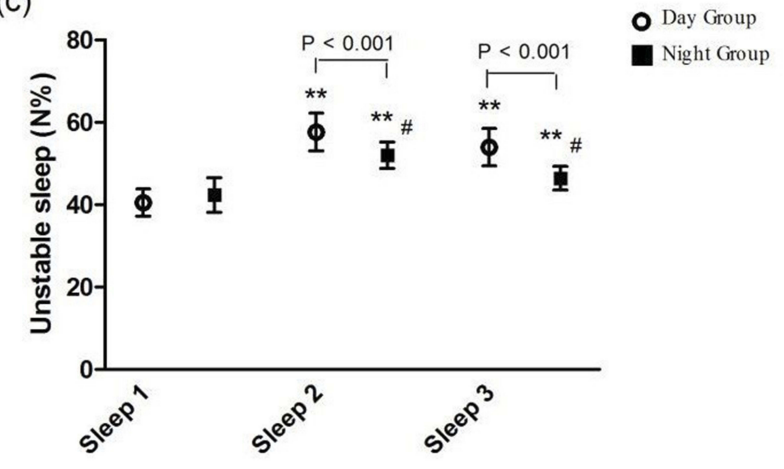

(b)

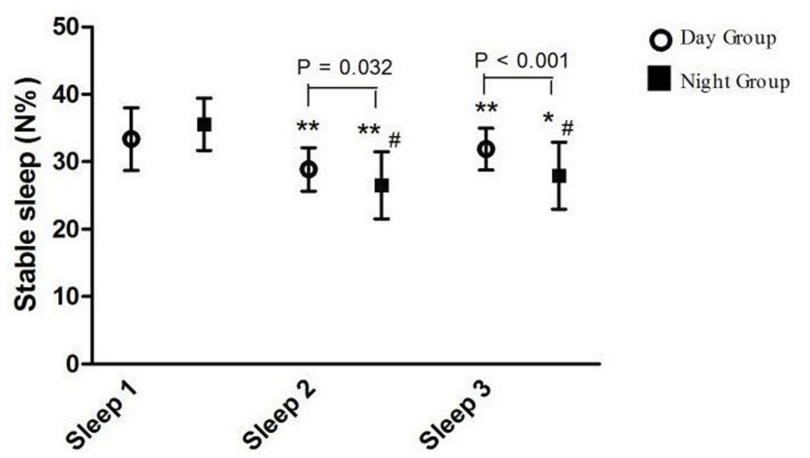

Figure 4 Comparison of the percentage of sleep stages distribution between the Day Group and the Night Group. (A) REM; (B) Stable sleep; (C) Unstable sleep. REM: Rapid eye movement; Sleep I: the night before surgery; Sleep 2: the first night after surgery; Sleep 3: the third night after surgery. In the same group, * vs Sleep I: $\mathrm{P}<0.05 ; * *$ vs Sleep I: $\mathrm{P}<0.001$. At the same point, \# vs the Day Group: $\mathrm{P}<0.05$.

\section{Postoperative VAS Evaluation}

After surgery, all patients received sufentanil-based PCA pump. Postoperative pain was assessed with VAS. During the first 48 hrs, patients in the $\mathrm{N}$ Group had a higher VAS score compared to the D Group at 1, 6, 24, $48 \mathrm{hrs}$ after surgery $(2.97 \pm 0.9,2.17 \pm 0.8,1.47 \pm 0.7$ and $1.07 \pm 0.5$ vs $2.60 \pm 1.0,1.77 \pm 0.6,0.87 \pm 0.6$ and $0.63 \pm 0.6, \mathrm{P}<0.05$, respectively) and a higher pump press numbers $(36.03$ \pm 2.1 vs $28.90 \pm 3.3, \mathrm{P}<0.05$ ) (Figure $5 \mathrm{~A}$ and $\mathrm{B}$ ).

\section{Discussion}

Our result confirmed that patients would suffer from obvious sleep disturbance after receiving general anesthesia. And the effect of the nighttime operation on 

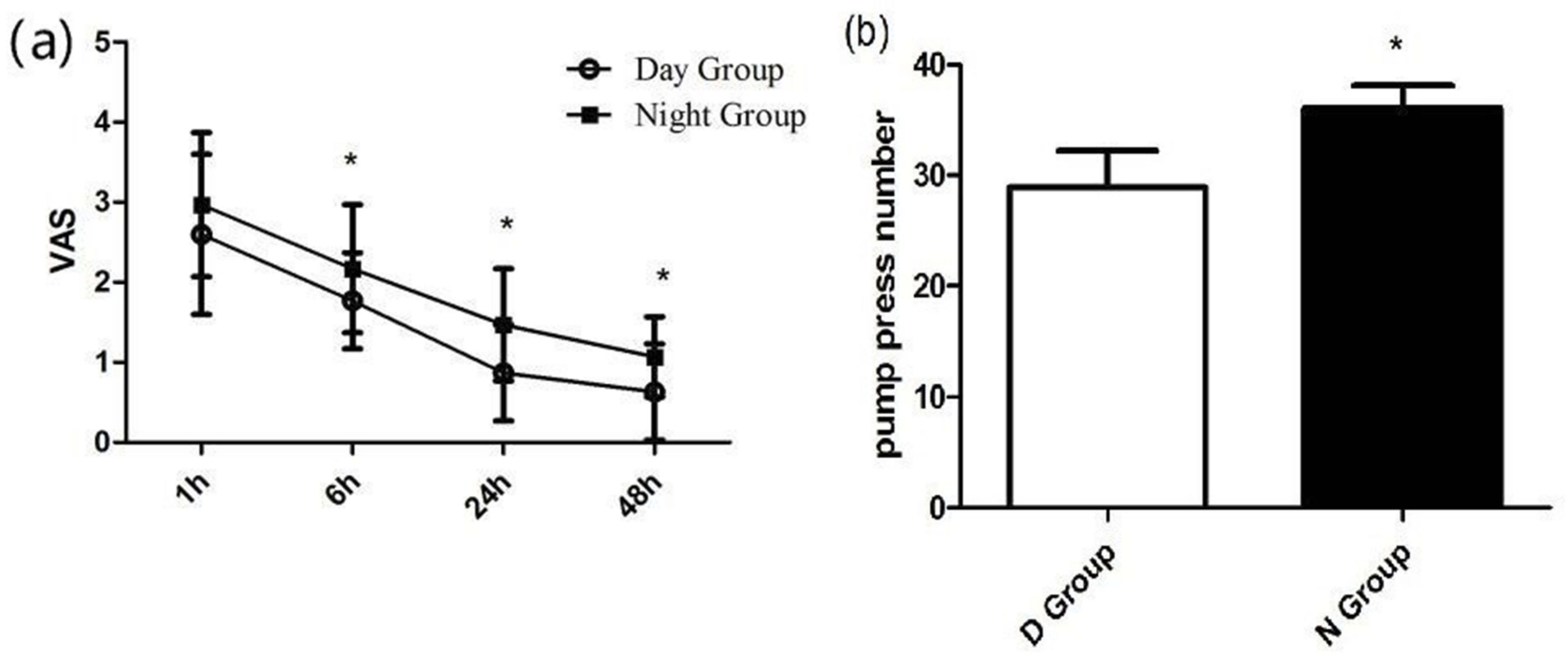

Figure 5 Postoperative VAS and PCA pump press numbers. (A) Postoperative VAS between the Day Group and the Night Group. (B) Postoperative PCA pump press number between the Day Group and the Night Group. VAS: Visual analogue scale * vs the Day Group: $\mathrm{P}<0.05$

postoperative sleep disturbance was more obvious than that of daytime operation. However, intraoperative use of DEX during the daytime operation could better improve the sleep efficiency and subjective sleep quality and promote the analgesia property of sufentanil-based PCA than intraoperative use of DEX during the nighttime operation.

In the current study, our data demonstrated that there was severe sleep disturbance with lower sleep efficiency and higher AIS score after surgery, which was manifested as decreased percentage of REM and Stable sleep. Moreover, it seems that the decrease of postoperative REM and Stable sleep after the nighttime operation were larger than those after the daytime operation and the extubation time at night was also longer than that during the day. This could be explained as the general anesthetics may be relatively overdose at night, which was consistent with Chassard D's study that depending on the time-of-day of administration, the doses and duration of action of general anesthetics during the day were different from that at night. ${ }^{13}$

Akeju et al have stated that pharmacological induction of biomimetic sleep may significantly reduce drug-induced neurocognitive dysfunction. ${ }^{14}$ Insight from these advances suggests that $\alpha 2$ adrenergic receptor agonist medications may closely pattern the activity of arousal nuclei similar to non-REM sleep. ${ }^{15}$ In our study, we found that the dosage of DEX in the D Group was significantly more than that in the N Group, and the Unstable sleep of patients in the D Group increased more obviously than patients in the $\mathrm{N}$
Group, which was likely associated with DEX's pharmacologic activation of the endogenous sleep-promoting pathway to produce a state resembling physiologic stage $\mathrm{N} 2$ sleep $^{16}$ and indicated its beneficial effect focusing on improving sleep quality and reducing sleep fragmentation. Meanwhile, the sleep efficiency and subjective sleep quality in the D Group were better than those in the N Group, which was consistent with the previous conclusion that though DEX has no improvement on REM and Stable sleep, it could prolong the total sleep time and ameliorate the sleep architecture by increasing Unstable sleep. ${ }^{8}$

Previous study found that patients sedated with DEX during the operation under spinal anesthesia may have experienced worse postoperative sleep. Their finding may because patients were awake during the operation, with the deeper sedative state provided by DEX, the natural sleep cycle of patients was disturbed in the daytime and then have worse effect on the night after surgery, which was different from our study that involved patients under general anesthesia. ${ }^{11}$

Previous study also indicated that postoperative sleep disturbance could lead to hyperalgesia. ${ }^{17}$ Effective postoperative pain relief might break the vicious cycle and have a positive effect on the recovery of postoperative sleep disturbance. Among the various available protocols for postoperative pain management, opioids are widely used. Some studies indicated that opioids also have negative impact on sleep through inhibition of REM and Stable sleep, which could aggravate the influence of postoperative sleep 
disorders on patients. ${ }^{18}$ Clinical studies have already confirmed that the potential of DEX as an adjuvant for pain treatment could contribute to the recovery-promoting effect following different kinds of operations and this use suggests that DEX might be a new drug for surgery-induced acute pain control. ${ }^{19-21}$ In the present study, we combined DEX with propofol and remifentanil to maintain the general anesthesia, and we found patients from the D Group had lower VAS score and less PCA pump press numbers than those in the $\mathrm{N}$ Group during the first $48 \mathrm{hrs}$ after surgery, which showed that intraoperative DEX was helpful in relieving postoperative pain and postoperative pain relief was more effective among patients receiving daytime operation than those receiving nighttime operation. And through administering a continuous infusion during the operation, we did not see a significant decrease in HR or MAP between the two groups, which were consistent with previous reports which indicated that intraoperative DEX provided more stable anesthesia without changing hemodynamic characteristics, and promoted quality of recovery from surgeries. ${ }^{22-24}$

There might be several limitations in the present study. First, only one preoperative night of monitoring is recorded in our design; the unusual situation might cause changes in the PSM. Second, though nocturnal interventions were reduced as much as possible, we could not completely rule out factors that might have negative effects on the quality of sleep such as light, noise or interruptions due to nursing care. Third, the patients enrolled in this study were 30-55 years old; the effect of DEX on other patients such as elderly patients or children should be further investigated. Fourth, this is a singlecenter analysis character focusing only on one specific hospital and one population, and multicenter experiment should be further studied.

\section{Conclusion}

Using DEX during the daytime operation could better improve postoperative sleep efficiency and subjective sleep quality and promote the analgesia property of sufentanil-based PCA than intraoperative use of DEX during the nighttime operation. Our study recommended that the intraoperative dose of DEX and the dose of postoperative analgesics for the nighttime operation should be increased appropriately compared with those for the daytime operation.

\section{Abbreviations}

Dexmedetomidine, DEX; Visual analog scale, VAS; The Portable Sleep Monitor, PSM; Rapid eye movement,
REM; Slow wave sleep, SWS; The Athens Insomnia Scale, AIS; Heart rate, HR; Mean arterial pressure, MAP; non-invasive blood pressure, NIBP; peripheral oxygen saturation, $\mathrm{SpO}_{2}$.

\section{Data Sharing Statement}

The individual deidentified participant data in our study could be shared with the readers. And readers can obtain the data by emailing the corresponding author (zhujunchao1@hotmail.com). We did not have specific data and other study-document in our study. All the data in our study are available for ten years.

\section{Acknowledgments}

The authors would like to thank Raymond C. Koehler, MD, Ph.D., from Departments of Anesthesiology and Critical Care Medicine, Johns Hopkins, University, Baltimore, MD, USA and Dr. Weifeng Song, MD, Ph.D., from Department of Anesthesiology and Perioperative Medicine, School of Medicine, the University of Alabama at Birmingham, Birmingham, Alabama, USA, for their discussion and advice of this study. The present study was funded by the Support Plan for Innovative Talents in Liaoning Higher Education Institution (grant no. 201834).

\section{Author Contributions}

All authors contributed to data analysis, drafting or revising the article, gave final approval of the version to be published, and agree to be accountable for all aspects of the work.

\section{Disclosure}

The authors report no conflicts of interest in this work.

\section{References}

1. Miller RD. Miller's Anesthesia. 7th ed. Churchill Livingstone Elsevier; 2010.

2. Krenk L, Jennum P, Kehlet H. Sleep disturbances after fast-track hip and knee arthroplasty. Br J Anaesth. 2012;109(5):769-775. doi:10.1093/bja/ aes 252

3. Masllow GA, Lipinski WJ, Matlen LB, et al. Isoflurane anesthesia does not satisfy the homeostatic need for rapid eye movement sleep. Anesth Analg. 2010;110:1283-1289. doi:10.1213/ANE.0b013e3181d3e861

4. Pick J, Chen Y, Moore JT, et al. Rapid eye movement sleep debt accrues in mice exposed to volatile anesthetics. Anesthesiology. 2011;115(4):702-712. doi:10.1097/ALN.0b013e31822ddd72

5. Su S, Ren C, Zhang H, Liu Z, Zhang Z. The opioid-sparing effect of perioperative DEX plus sufentanil infusion during neurosurgery: a retrospective study. Front Pharmacol. 2016;7:407. doi:10.3389/ fphar.2016.00323 
6. Mohta M, Kalra B, Sethi AK, Kaur N. Efficacy of DEX as an adjuvant in paravertebral block in breast cancer surgery. J Anesth. 2016;30(2):252-260. doi:10.1007/s00540-015-2123-8

7. Kim SH, Oh YJ, Park BW, Sim J, Choi YS. Effects of single-dose DEX on the quality of recovery after modified radical mastectomy: a randomised controlled trial. Minerva Anestesiol. 2013;79:1248-1258.

8. Wu XH, Cui F, Zhang C, et al. Low-dose dexmedetomidine improves sleep quality pattern in elderly patients after noncardiac surgery in the intensive care unit: a pilot randomized controlled trial. Anesthesiology. 2016;125(5):979-991. doi:10.1097/ALN.0000000000001325

9. Djaiani G, Silverton N, Fedorko L, et al. Dexmedetomidine versus propofol sedation reduces delirium after cardiac surgery: a randomized controlled trial. Anesthesiology. 2016;124:362-368. doi:10.1097/ALN.0000000000000951

10. Zhang H, Lu Y, Liu M, et al. Strategies for prevention of postoperative delirium: a systematic review and meta-analysis of randomized trials. Crit Care. 2013;18(17):R47.

11. Tan W, Miao E, Jin F, Hong M, Huangwei L. Changes in first postoperative night bispectral index after daytime sedation induced by dexmedetomidine or midazolam under regional anesthesia a randomized controlled trial. Reg Anesth Pain Med. 2016;41(3):380-386. doi:10.1097/AAP.0000000000000370

12. El Sherif FA, Othman AH, Abd ER, Ahmad M, Taha O. Effect of adding intrathecal morphine to a multimodal analgesic regimen for postoperative pain management after laparoscopic bariatric surgery: a prospective, double-blind, randomized controlled trial. $\mathrm{Br} J$ Pain. 2016;10(4):209-216. doi:10.1177/2049463716668904

13. Chassard D, Bruguerolle B. Chronobiology and anesthesia. Anesthesiology. 2004;100(2):413-427. doi:10.1097/00000542-20040 2000-00034

14. Akeju O, Brown EN. Neural oscillations demonstrate that general anesthesia and sedative states are neurophysiologically distinct from sleep. Curr Opin Neurobiol. 2017;44:178-185. doi:10.1016/j. conb.2017.04.011

15. Weber F, Dan Y. Circuit-based interrogation of sleep control. Nature. 2016;538:51-59. doi:10.1038/nature19773
16. Huupponen E, Maksimow A, Lapinlampi P, et al. Electroencephalogram spindle activity during dexmedetomidine sedation and physiological sleep. Acta Anaesthesiol Scand. 2008;52(2):289-294. doi:10.1111/ j.1399-6576.2007.01537.x

17. Wang PK, Cao J, Wang H, et al. Short-term sleep disturbanceinduced stress does not affect basal pain perception, but does delay postsurgical pain recovery. J Pain. 2015;16(11):1186-1199. doi:10.1016/j.jpain.2015.07.006

18. Chouchou F, Khoury S, Chauny JM, Denis R, Lavigne GJ. Postoperative sleep disruptions: a potential catalyst of acute pain? (Review). Sleep Med Rev. 2014;18(3):253-262. doi:10.1016/j.smrv.2013.07.002

19. Movafegh A, Shoeibi G, Ansari M, Sadeghi M. Naloxone infusion and post-hysterectomy morphine consumption: a double-blind, placebo-controlled study. Acta Anaesthesiol Scand. 2012;56(10):12411249. doi:10.1111/j.1399-6576.2012.02764.x

20. Zhao JN, Kong M, Qi B, Ge DJ. Comparison of the morphinesparing effect of intraoperative DEX with and without loading dose following general anesthesia in multiple-fracture patients: a prospective, randomized, controlled clinical trial. Medicine (Baltimore). 2016;95:e4576. doi:10.1097/MD.0000000000004864

21. Ge DJ, Qi B, Tang G, Li JY. Intraoperative DEX promotes postoperative analgesia and recovery in patients after abdominal hysterectomy: a double-blind, randomized clinical trial. Sci Rep. 2016;6:21514. doi:10.1038/srep21514

22. Bekker A, Haile M, Kline R, et al. The effect of intraoperative infusion of dexmedetomidine on the quality of recovery after major spinal surgery. $J$ Neurosurg Anesthesiol. 2013;25(1):16-24. doi:10.1097/ANA.0b013e31826318af

23. Kim SY, Kim JM, Lee JH, Song BM, Koo BN. Efficacy of intraoperative dexmedetomidine infusion on emergence agitation and quality of recovery after nasal surgery. Br J Anaesth. 2013;111(2):222228. doi:10.1093/bja/aet056

24. Chattopadhyay U, Mallik S, Ghosh S, Bhattacharya S, Bisai S, Biswas $\mathrm{H}$. Comparison between propofol and dexmedetomidine on depth of anesthesia: a prospective randomized trial. J Anaesthesiol Clin Pharmacol. 2014;30(4):550-554. doi:10.4103/0970-9185.142857

\section{Publish your work in this journal}

Nature and Science of Sleep is an international, peer-reviewed, open access journal covering all aspects of sleep science and sleep medicine, including the neurophysiology and functions of sleep, the genetics of sleep, sleep and society, biological rhythms, dreaming, sleep disorders and therapy, and strategies to optimize healthy sleep.
The manuscript management system is completely online and includes a very quick and fair peer-review system, which is all easy to use. Visit http://www.dovepress.com/testimonials.php to read real quotes from published authors. 